\title{
Un modèle évolutionnaire de segmentation des marchés du vin
}

Politiques de qualité et caractéristiques informationnelles

An evolutionary model of the wine markets segmentation: quality policies and signals

\section{Christian Barrère}

\section{(2) OpenEdition}

\section{Journals}

Édition électronique

URL : http://journals.openedition.org/economierurale/1902

DOI : 10.4000/economierurale.1902

ISSN : 2105-2581

Éditeur

Société Française d'Économie Rurale (SFER)

Édition imprimée

Date de publication : 15 novembre 2007

Pagination : 18-35

ISSN : 0013-0559

Référence électronique

Christian Barrère, "Un modèle évolutionnaire de segmentation des marchés du vin », Économie rurale [En ligne], 301 | Septembre-octobre 2007, mis en ligne le 15 novembre 2009, consulté le 19 avril 2019 URL : http://journals.openedition.org/economierurale/1902; DOI : 10.4000/economierurale.1902 


\title{
Un modèle évolutionnaire de segmentation des marchés du vin Politiques de qualité et caractéristiques informationnelles
}

\author{
Christian BARRÈRE • Université de Reims, Organisations marchandes et institutions, OMI
}

es marchés des vins sont aujourd'hui entrés -dans une période de mutations qui entraîne, pour certains vignobles, des crises fortes. De nouveaux producteurs et de nouveaux consommateurs ont pris une place conséquente sur les marchés mondiaux. Dans les vieux pays vitivinicoles, les producteurs de vins de qualité prennent leurs distances avec des stratégies d'appellation qui se sont généralisées à un point tel qu'elles perdent de leur pouvoir signifiant et ils s'engagent dans des politiques de marque plus affirmées. L'organisation traditionnelle des marchés et les mécanismes de régulation offre-demande et de détermination des quantités et de qualité correspondantes perdent de leur efficacité. Un des aspects essentiels des difficultés et des mutations en cours nous semble résider dans la remise en cause de la segmentation sur laquelle ils reposaient et que nous appellerons la segmentation aristocratique.

Les marchés des vins ont en effet obéi pendant des siècles à une segmentation forte et relativement constante et ce jusqu'à la fin du $\mathrm{XX}^{\mathrm{e}}$ siècle : d'un côté des vins de qualité élevée et régulière, vendus sur des marchés locaux et internationaux, de l'autre des vins de qualité courante destinés à la consommation populaire ; d'un côté, de façon dominante, des stratégies de qualité, de l'autre, de façon dominante, des stratégies de quantité. Rendre compte des problèmes actuels c'est donc s'interroger sur l'évolution de cette segmentation et, en premier lieu, sur la logique économique de la segmentation aristocratique et son adéquation aux conditions actuelles de l'offre, de la demande et de l'échange sur des marchés désormais globalisés.
Pour cela il faut disposer d'instruments d'analyse des stratégies de qualité menées par les offreurs et de leur résultante sur le fonctionnement du marché. Depuis les travaux fondateurs de Chamberlin sur la différenciation (Chamberlin, 1933), de Lancaster sur les biens à caractéristiques (Lancaster, 1966), d'Akerlof sur les signaux de qualité (Akerlof, 1970), l'analyse de la qualité a pris une place importante dans l'analyse économique, en particulier en liaison avec la considération de l'imperfection de l'information. Cependant elle n'a pas encore débouché sur une théorie acceptée de la détermination concurrentielle des niveaux de qualité sur un marché particulier. Nous nous proposons de participer à cet effort en modélisant les situations de détermination stratégique de la qualité sur des marchés du type des marchés aristocratiques traditionnels. Un tel modèle nous semble capable de définir les sentiers possibles d'évolution sur ces marchés, en fonction de déterminants synthétiques. Il ne suffit évidemment pas à rendre compte de la dynamique d'ensemble des marchés mondiaux des vins mais ce modèle peut constituer un cadre de raisonnement pour étudier l'effet de changements déterminés sur l'équilibre évolutionnaire des marchés aristocratiques et, particulièrement, sur la poursuite, l'effacement ou la recomposition de la segmentation aristocratique.

La qualité proposée par les offreurs dépend en effet de choix effectués dans des situations d'interdépendance stratégique, c'est-à-dire quand différents offreurs sont en concurrence au moyen de politiques de qualité dont les résultats dépendent de la 
dialectique de l'ensemble de leurs choix. Ces résultats ne sont pas triviaux et peuvent même être contre-intuitifs. Pour en rendre compte, la théorie économique dispose, pour le moment au moins, d'un seul instrument, la théorie des jeux, mais d'un instrument d'autant plus puissant qu'il a connu depuis la guerre des enrichissements substantiels et continus. Le fonctionnement du marché peut être considéré comme le déroulement d'un jeu dans lequel des acteurs (des joueurs) usent de stratégies pour obtenir des gains dont ils savent qu'ils dépendent in fine de la résultante de l'interaction de tous les choix stratégiques. Nous nous référerons plus spécifiquement à une approche en termes de jeux évolutionnaires puisque notre problème est celui de l'évolution, à travers le temps, avec des acteurs changeants, en nombre important, au milieu d'aléas, et compte tenu des résultats temporaires de l'interaction des stratégies, de la segmentation des marchés du vin. La théorie des jeux évolutionnaires ${ }^{1}$ définit en effet des équilibres évolutionnaires - des Equilibres évolutionnairement stables (EES) - situations dans lesquelles tous les joueurs utilisent des Stratégies (pures ou mixtes) évolutionnairement stables (SES), stratégies capables de résister à l'invasion de stratégies alternatives (Weibull, 1995). Elle suppose que les stratégies qui donnent les meilleurs gains sont progressivement préférées par les joueurs. De ce fait il n'est pas besoin de poser des hypothèses fortes de rationalité substantive (qui, dans le cas des jeux stratégiques habituels, requièrent que les joueurs soient capables d'anticiper les stratégies des autres sur l'ensemble du déroulement du jeu). Les joueurs peuvent se contenter d'observer le résultat du jeu et s'aligner, graduellement, via un processus élémentaire d'apprentissage, sur les stratégies gagnantes ; parallèlement, les erreurs, les mutations, l'entrée de nouveaux joueurs porteurs de nouvelles stratégies sont possibles.

1. Cf. Annexe 1.
Nous utilisons alors une démarche hypothético-déductive en construisant un modèle général sur la base d'hypothèses très simples correspondant à l'existence de marchés aristocratiques (les producteurs peuvent choisir des politiques de qualité mais n'y ont pas forcément toujours intérêt). Nous nous interrogeons ensuite sur les conditions nécessaires pour que la segmentation aristocratique se maintienne, c'est-à-dire pour que les marchés des vins aristocratiques continuent à fournir de la qualité élevée, les séparant des autres marchés. Le modèle permet de définir toutes les variantes formellement envisageables et les sentiers d'évolution correspondants, en fonction de la relation qui s'établit entre deux déterminants synthétiques précis (appelés prime d'alignement stratégique sur la qualité haute et prime d'alignement stratégique sur la qualité basse). Ces déterminants expriment les conditions régnantes sur le marché, conditions qui sont de trois ordres : des conditions relatives à l'offre (coûts, compétitivité, etc.), des conditions relatives à la demande (préférences, pouvoirs d'achat, ...), des conditions relatives à l'échange (en particulier en matière d'information - pour qu'un choix de qualité haute l'emporte sur un choix de qualité basse, encore faut-il que le producteur puisse signaler sa qualité). Dans ce texte nous laisserons de côté le problème des conditions de production en supposant que, pour les marchés qui nous intéressent, il est possible de produire du vin de qualité à un coût correspondant aux dispositions à payer de la fraction aristocratique des consommateurs, mais que les conditions institutionnelles de l'information n'assurent pas nécessairement le triomphe de ces stratégies de qualité élevée sur des stratégies de qualité faible. Nous nous intéresserons donc au lien entre stratégies de qualité et conditions d'information. En précisant les divers types de configurations auxquelles peut aboutir le jeu stratégique concurrentiel c'est-à-dire l'ensemble du spectre des possibles, le modèle nous permet de nous interroger enfin 
sur les conditions institutionnelles réelles nécessaires pour la reproduction de la segmentation traditionnelle des marchés des vins et donc sur sa vraisemblance.

La première partie précisera le point de départ de l'analyse, les notions de paradigme aristocratique du goût et de segmentation aristocratique des marchés. Le modèle de détermination stratégique de la qualité sur des marchés aristocratiques traditionnels sera présenté dans la deuxième partie. Quatre configurations, qui peuvent formellement se manifester, seront étudiées dans la troisième partie : si l'une d'entre elles assure spontanément la reproduction de la segmentation aristocratique, les trois autres appellent des régulations institutionnelles faute de quoi dominent des sentiers d'évolution conduisant les producteurs à quitter le segment aristocratique. L'on dispose alors d'un modèle susceptible d'éclairer l'effet des changements intervenant dans l'offre et la demande de vin sur les stratégies des acteurs et la segmentation qui en découlait. La conclusion de cette analyse s'ouvre sur de nouvelles perspectives.

\section{L'hypothèse d'un paradigme aristocratique du goût}

Nous parlons de segmentation aristocratique parce que nous l'interprétons comme l'expression d'un paradigme aristocratique du goût. L'idée de paradigme du goût résulte de la prise en compte des spécificités des biens de goût tels que le vin. En premier lieu, ceux-ci ont de fortes caractéristiques sémiotiques. Au-delà de caractéristiques matérielles, ils expriment des signifiants, véhiculent des images, des symboles, des représentations. La demande de biens alimentaires et gastronomiques n'est que pour partie demande de nourriture mais est aussi - et dans certains cas d'abord demande de signes et de sens. La demande de Champagne est indissociable de l'image de ce vin comme vin de la fête et du luxe. En deuxième lieu, les biens de goût incluent au- delà de leurs caractéristiques naturelles des caractéristiques culturelles. La cuisine est un fait culturel global, ce qui est du domaine du comestible et du non-comestible dépend des cultures considérées, les modes d'élaboration culinaires aussi (Lévi-Strauss, 1964 ; Fischler, 1993). La proportion entre consommation de vin blanc et de vin rouge dans différents pays européens ne dépend pas tant des prix relatifs de ces vins que d'habitudes de consommation et de patrimoines culturels. Enfin ces biens incorporent des caractéristiques relationnelles. Leur consommation ne se limite pas à une relation bien-individu mais est fonction des relations entre l'individu et les autres individus ou les groupes, comme cela a été mis en évidence pour la première fois dans le cas d'école de la mode (Simmel, 1904 ; Veblen, 1899 ; Spencer, 1854). Ces caractéristiques ont-elles un effet sur les conditions de formation des demandes de biens de goût ?

Les demandes de biens de goût dépendent, comme celles de tout bien, des prix de ces biens (et de ceux de leurs substituts et compléments), des revenus et richesses des consommateurs et de leurs goûts et préférences. La théorie économique propose habituellement de privilégier les variables de prix et revenu, renvoyant l'analyse des goûts et préférences à des disciplines extérieures au champ de la science économique (la psychologie et la psychologie sociale, la sociologie, la psychanalyse, ...), et donc les posant comme des données, selon l'adage beckérien De gustibus non est disputandum (Becker, 1996). Certes l'évolution des prix contribue à expliquer les évolutions des demandes de biens de goût. Elle n'explique pourtant qu'une partie de celles-ci et pas nécessairement la plus grande. Dans de très nombreux cas ce sont les modifications des préférences et goûts, sous l'effet de changements sociaux ou de stratégies des offreurs, qui sont déterminantes. De même les différences spatiales ou historiques des consommations de biens de goût sont-elles conditionnées par des systèmes de préfé- 
rences différenciés. Nous avons, à partir de l'étude du secteur de la mode (Barrère et Santagata, 2005), avancé l'idée selon laquelle l'évolution des conditions de formation des goûts et des demandes pouvait être utilement éclairée par l'hypothèse de la succession de différents paradigmes du goût.

Un paradigme du goût désigne l'ensemble des questions et réponses qu'une société donnée avance pour définir le statut socioéconomique du goût : qu'est-ce qui est de bon goût ou non, qui définit le goût - en matière de mode on parlera des " arbitres des élégances »-, quels sont les objets pour lesquels la notion de goût a un sens... ? Un paradigme du goût s'inscrit dans le cadre d'un paradigme sociétal, c'est-à-dire de la façon dont la société, à une époque historique donnée, se représente - ou du moins dans la façon dont le consensus dominant la représente -, définit la place des groupes et individus, organise les rapports de pouvoir...

Le paradigme sociétal aristocratique représente la société comme composition de deux groupes hétérogènes et étanches, l'élite et le peuple. Il existerait une élite sociale dirigeante, séparée du peuple, radicalement distincte de celui-ci - les uns ont du sang bleu quand les autres n'en ont que du rouge -, figée et protégée par l'absence de canaux de mobilité. Le paradigme aristocratique du goût, qui s'inscrit dans cette vision globale, s'organise autour de l'idée que le goût est propre à l'élite. De ce fait, toute une série de biens de goût sera réservée à l'aristocratie. Il en est ainsi des biens de luxe conçus comme biens spécifiques de la consommation aristocratique - la « Haute Couture », la « Grande Cuisine » (chez Carême, deux tiers des plats présentés ont des noms évocateurs de la noblesse, par exemple « lièvre à la royale », ...), les « Grands Vins ». Biens de goût aristocratique et biens de goût populaire sont ainsi totalement disjoints. On connaît la distinction opérée par Bourdieu entre « goûts de luxe » et « goûts de nécessité » : les classes populaires ont peu de choix, leur consommation est du domaine de la nécessité et elles l'auto-justifient en «faisant de nécessité vertu » alors que le goût de luxe est marqué par la distance à la nécessité (Bourdieu, 1979). L'aristocratie est d'ailleurs souvent désignée comme les "gens de qualité », les « gens de goût » (cf. le ridicule dont est frappé le Bourgeois Gentilhomme, symbole du mauvais goût parce qu'il usurpe un goût qui ne correspond pas à sa condition). Le vulgaire ne saurait apprécier les biens de goût : autant « donner de la confiture à des cochons ». La disjonction entre goût aristocratique et goût(s) populaire(s) constitue le fondement de ce modèle caractérisable comme «modèle disjonctif » puisque reposant sur la différence de statut entre biens aristocratiques et biens populaires. Cela prédétermine deux types de fonction de demandes, radicalement séparées, celles de l'aristocratie, celles du peuple, et segmente les goûts et préférences selon la même frontière. Le paradigme aristocratique conduit les consommateurs à s'auto-sélectionner et à se ranger dans le type de demande que leur autorise leur statut social.

Dans ce contexte s'est constituée et rigidifiée une segmentation entre vins aristocratiques et vins populaires, qui a souvent, en France notamment, pris la forme d'une segmentation territoriale des vignobles. Cependant, des changements significatifs sont récemment intervenus, à la fois dans l'offre et dans la demande de vin, à l'échelle internationale. Vont-ils remettre en cause la segmentation traditionnelle, conduire à la disparition ou à la réorganisation des marchés aristocratiques du vin ?

\section{Un modèle évolutionnaire de détermination stratégique de la qualité}

Dans le modèle des vins aristocratiques, caractérisé par une très forte différenciation horizontale et verticale, d'autant plus efficace que la consommation aristocratique est le fait d'un cercle restreint de consommateurs éclairés, chaque région viticole a sa propre autonomie, fondée sur les spécificités de 
terroir, de cépage, de tradition d'élevage et d'assemblage. Les spécificités peuvent aller jusqu'à constituer en marchés séparés des vins particuliers. Définir les limites du marché pertinent n'est pas toujours chose facile et n'entre pas dans l'objet de cet article. Nous supposerons que le marché ou le segment de marché considéré peut être représenté par un jeu particulier².

Le point de départ du jeu est l'existence d'une qualité haute et d'une forte réputation, corollaires de la consommation aristocratique. Cependant, la stratégie de qualité haute peut se voir contestée par une stratégie opportuniste de baisse de la qualité, qui donne du profit en économisant sur les coûts, voire en élargissant le marché grâce à la baisse des coûts et des prix. Rien ne garantit donc a priori la reproduction du niveau de qualité, qui dépend du déroulement d'un jeu entre de nombreux joueurs, donc de la dialectique de leurs choix stratégiques. Chacun peut choisir un niveau élevé ou bas de qualité (les deux stratégies pures du jeu) ou n'importe quel niveau intermédiaire (les stratégies mixtes). L'on notera $\mathrm{h}$ pour les joueurs (1) et $\mathrm{H}$ pour les joueurs (2) la stratégie pure de qualité haute, $\mathrm{b}$ et $\mathrm{B}$ celle de qualité basse. Le jeu se présente alors, sous sa forme stratégique et sa forme stratégique normalisée comme suit :

\section{H B}
(1)

$\begin{array}{lll}\text { h } & \alpha, \alpha^{\prime} & \beta, \beta \\ \text { b } & \gamma, \gamma^{\prime} & \delta, \delta\end{array}$
b $\gamma, \gamma$
$\delta, \delta$

$a_{1}$ représente la différence de gain des joueurs (1) quand ils répondent à la stratégie $\mathrm{H}$ des joueurs (2) par $\mathrm{h}$ au lieu de $\mathrm{b} ; \mathrm{a}_{2}$ la différence de gain des joueurs (1) quand ils répondent à la stratégie $\mathrm{B}$ des joueurs (2) par $\mathrm{b}$ au lieu de $\mathrm{h} ; \mathrm{b}_{1}$ la différence de gain des joueurs (2) quand ils répondent à la stratégie $\mathrm{h}$ des joueurs (1) par $\mathrm{H}$ au lieu de B ; $\mathrm{b}_{2}$ la différence de gain des joueurs (2) quand ils répondent à la stratégie b des joueurs (1) par B au lieu de $\mathrm{H}$.

Si la structure des gains est la même, c'est-à-dire si l'ordre des gains est le même pour $\alpha, \beta, \gamma, \delta$ et pour $\alpha^{\prime}, \beta^{\prime}, \gamma^{\prime}, \delta$ ', le jeu devient un jeu symétrique ${ }^{3}$. À ce moment-là, sur chaque marché il y a diversité des producteurs mais elle n'est pas suffisante pour conduire à des classements différents des gains et donc à des choix stratégiques différents dans les mêmes situations.

\begin{tabular}{|c|c|c|c|}
\hline & & & \\
\hline & & $\mathbf{H}$ & B \\
\hline (1) & $\mathbf{h}$ & $a_{1}$ & 0 \\
\hline & b & 0 & $\mathrm{a}_{2}$ \\
\hline
\end{tabular}

La résolution du modèle (par calcul des stratégies évolutionnairement stables et détermination des équilibres évolutionnairement stables) nous donne quatre configurations en fonction de la structure de la matrice des gains. Ces configurations ne dépendent que de deux facteurs, $a_{1}$ et $a_{2}$ et même de leurs seuls signes :

- $\mathrm{a}_{1}=(\alpha-\gamma)=\mathrm{x}\left(\alpha^{\prime}-\gamma^{\prime}\right)=\mathrm{G}(\mathrm{h}, \mathrm{H})-\mathrm{G}(\mathrm{b}, \mathrm{H})$; nous l'appellerons la prime d'alignement stratégique sur la qualité haute : elle représente la différence de gain entre les

\begin{tabular}{|cccc|}
\hline & & $\mathbf{H}$ & $(2)$ \\
(1) & $\mathbf{h}$ & $\alpha-\gamma=\mathrm{a}_{1} ; \alpha^{\prime}-\beta^{\prime}=\mathrm{b}_{1}$ & $\mathbf{B}$ \\
& $\mathbf{b}$ & 0,0 & 0,0 \\
& & $\delta-\beta=\mathrm{a}_{2} ; \delta^{\prime}-\gamma^{\prime}=\mathrm{b}_{2}$ \\
\hline
\end{tabular}

2. Cela n'empêche pas, dans un second temps, d'introduire des phénomènes de substitution et des interdépendances entre les différents segments.
3. Pour une explicitation de l'hypothèse de symétrie, cf. l'Annexe 2. 
réponses $\mathrm{h}$ et $\mathrm{b}$ quand les autres joueurs choisissent $\mathrm{H} ; \mathrm{a}_{1}>0$ signifie que $\mathrm{h}$ est la meilleure réponse à $\mathrm{H}, \mathrm{a}_{1}<0$ que c'est $\mathrm{b}$. - $\mathrm{a}_{2}=(\delta-\beta)=\mathrm{y}\left(\delta^{\prime}-\beta^{\prime}\right)=\mathrm{G}(\mathrm{b}, \mathrm{B})-\mathrm{G}(\mathrm{h}, \mathrm{B})$; nous l'appellerons la prime d'alignement stratégique sur la qualité basse : elle représente la différence de gain entre les réponses $h$ et $b$ au choix $B$ des autres joueurs $; a_{2}>0$ signifie que $b$ est la meilleure réponse à $\mathrm{B}, \mathrm{a}_{2}<0$ que c'est $\mathrm{h}$.

Quand les producteurs sont homogènes (leurs différences ne sont pas significatives et ne conduisent pas à des choix différents dans les mêmes situations), les différentes configurations avec leurs équilibres et leurs stratégies évolutionnairement stables sont représentées dans le graphique 1.

Les quatre configurations aboutissent à différentes issues avec des équilibres très variés.

Dans la configuration I nous avons une qualité haute évolutionnairement stable, chacun jouant une stratégie de qualité haute.

Dans la configuration II il y a deux équilibres, un équilibre haut et un bas, tous les joueurs choisissant au même moment la même stratégie; la probabilité de l'équilibre haut (ou l'étendue de son bassin d'attraction) dépend de $\mathrm{a}_{1} / \mathrm{a}_{2}$.

Dans la configuration III l'unique équilibre est un équilibre bas, tous les producteurs via l'observation des gains et l'apprentissage adoptent la stratégie de basse qualité.

Dans la configuration IV la solution est unique avec une qualité intermédiaire $\lambda$ $\left(\lambda=\mathrm{a}_{2} / \mathrm{a}_{1}+\mathrm{a}_{2}\right)$.

Si la population est hétérogène et se divise en deux sous-populations, les résultats sont identiques, excepté pour la configuration IV (nous étudierons ce cas plus loin).

Nous pouvons maintenant utiliser le modèle général pour décrire les divers sentiers d'évolution sur les marchés aristocratiques des vins en nous interrogeant sur les condi-

\section{Graphique 1. Les quatre configurations}

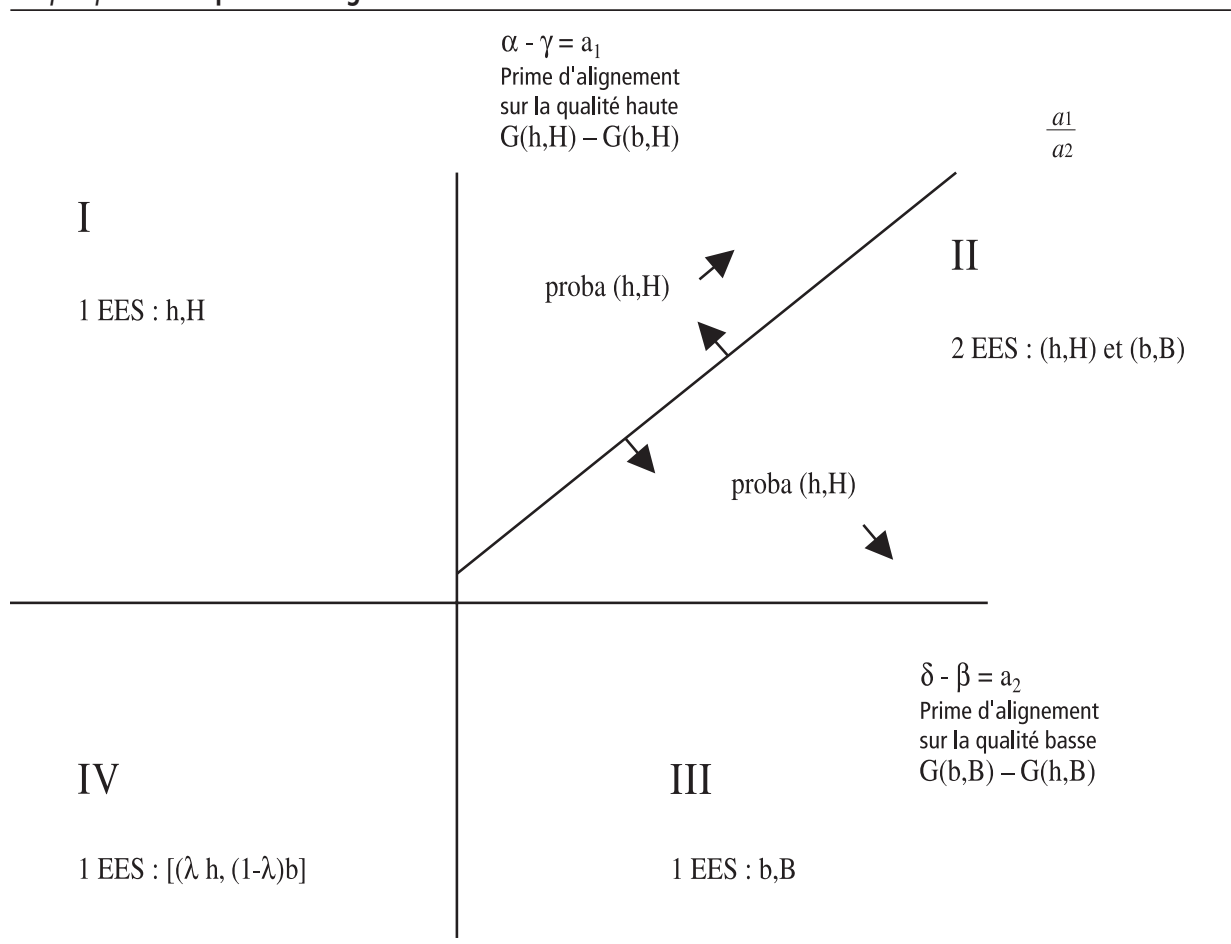

Source : conception de l'auteur. 
tions économiques qu'expriment les valeurs de $\mathrm{a}_{1}$ et $\mathrm{a}_{2}$. La connaissance de ces sentiers est le préalable à la compréhension des conditions de la segmentation qui assure la reproduction de marchés aristocratiques largement déconnectés des marchés populaires mais aussi à celle de ses limites. Pour chaque configuration nous définirons d'abord les conditions formelles auxquelles elle correspond; nous nous demanderons ensuite ce qu'elles signifient et impliquent en termes de conditions d'information et, éventuellement, de demande ; nous examinerons enfin la vraisemblance réelle d'une telle configuration en nous demandant si une telle configuration, formellement possible en fonction des valeurs de $\mathrm{a}_{1}$ et $\mathrm{a}_{2}$, peut correspondre à des marchés aristocratiques du vin.

\section{Les quatre configurations et les sentiers de développement}

\section{L'équilibre haut autorégulé : la configuration I et le sentier associé}

Le premier cas formellement possible est celui de la configuration $\mathrm{I}: \mathrm{a}_{1}>0$ et $\mathrm{a}_{2}<0$; $\mathrm{h}=\mathrm{MR}_{1}(\mathrm{H})$ et $\mathrm{h}=\mathrm{MR}_{1}(\mathrm{~B}), \mathrm{h}$ constitue la Meilleure Réponse à $(\mathrm{H})$ et à $(\mathrm{B})$, les producteurs choisissent tous la qualité haute ; le seul équilibre évolutionnairement stable est (h,H), équilibre robuste en stratégies dominantes, avec stabilité asymptotique. C'est la seule configuration qui reproduise de façon endogène la qualité d'un vin aristocratique en assurant une qualité haute évolutionnairement stable.

Un tel marché fonctionne comme un marché parfaitement informé. Pour que la condition $1, \mathrm{a}_{1}>0$ puisse se réaliser, la prime d'alignement sur la qualité haute être positive, il faut qu'il soit possible de produire de façon rentable un bien de qualité objet d'une demande régulière, mais aussi que les conditions d'information permettent de discriminer entre les producteurs, par exemple via l'existence de marques. Alors, les producteurs peuvent vendre de la bonne qualité, à coût plus élevé, mais à prix également plus élevé et, inversement, les consommateurs potentiels peuvent sanctionner les producteurs opportunistes. Pour que la condition $2, a_{2}<0$, s'établisse, que la prime d'alignement sur la qualité basse soit négative, il faut aussi que le vendeur de qualité puisse signaler sa spécificité.

Un tel type de marché du vin est traditionnellement défini comme un marché de connaisseurs. Il représente correctement les marchés des vins issus de vignobles aristocratiques sur lesquels tout le monde a intérêt à vendre de la haute qualité, plus rentable, et pour lesquels les conditions d'information le permettent. Vont en ce sens les caractéristiques institutionnelles suivantes :

1. Quand une variété est produite par un seul producteur (Petrus, Romanée Conti, ...) ou par un petit nombre de producteurs, les consommateurs connaissent les caractéristiques (particulièrement la qualité) de chaque offre et les distinguent en fonction du nom du producteur ou du terroir (par exemple Château Latour).

2. Au sein de l'aristocratie, la connaissance relative aux vins est reproduite par l'éducation et la culture, comme pour l'ensemble des produits créatifs (Caves, 2000), les échanges intellectuels portant sur le vin n'étant pas nécessairement porteurs de coûts de transaction ou d'information mais procurant plutôt des aménités.

3. La connaissance des vins est un élément de concurrence sociale pour les membres de l'aristocratie, conformément à la logique de la « société de Cour» (Elias, 1973).

4. Chaque marché est étroit et inclut un petit nombre seulement de producteurs.

5. Cérémonies et fêtes sont occasion de goûter les vins et d'en discuter entre connaisseurs.

Ces caractéristiques sociales étant données, l'institution adéquate au bon fonctionnement du marché est celle qui produit l'information : c'est « l'étiquette », à savoir le nom du terroir ou celui du producteur. 
C'est un système plus proche de celui de la griffe du grand couturier que de la marque. Le sentier de développement de ce type de configuration (le sentier 1) est l'autoreproduction de la qualité haute, comme il en a été historiquement ainsi pour les grands vins de Bourgogne et de Bordeaux. Cependant, ces conditions sont rarement observées et plutôt seulement dans le cas des grands vins, dont les qualités et les prix justifient l'effort d'évaluation des connaisseurs. En revanche les coûts relatifs de transaction et d'information concernant nombre de vins de qualité moyenne sont trop élevés pour que cette configuration les représente. L'existence des trois autres types de configuration, dans lesquels l'information est imparfaite, met alors en évidence les difficultés de la reproduction régulière d'une qualité élevée.

\section{Les équilibres bas : les configurations II et III et les deux sentiers correspondants}

Avec les configurations II et III nous quittons les marchés sur lesquels les conditions d'information sont proches du modèle théorique de marché parfait.

\section{La configuration III et le sentier 2a de sortie}

La configuration III correspond à $\mathrm{a}_{1}<0$ et $\mathrm{a}_{2}>0 ; 0 ; \mathrm{b}=\mathrm{MR}(\mathrm{H})$ et $\mathrm{b}=\mathrm{MR}(\mathrm{B}), \mathrm{b}$ constitue la Meilleure Réponse à $(\mathrm{H})$ et à (B). Dans ces conditions, la politique de qualité disparaît dans un dilemme du prisonnier avec un équilibre sous-optimal robuste puisqu'en stratégies dominantes $(b, B)$ est le seul équilibre évolutionnairement stable (EES), B étant la seule stratégie évolutionnairement stable (SES) 4

L'imperfection de l'information peut parfaitement expliquer que ces conditions se réalisent. Elle permet d'abord que la stratégie de qualité basse soit la meilleure

4. Un mécanisme de réplication standard (Weibull, 1995, chapitres 3 et 5) donne la dynamique: $\mathrm{dh} / \mathrm{dt}=\mathrm{h}(1-\mathrm{h})\left[\mathrm{a}_{1} \mathrm{~h}-\mathrm{a}_{2}(1-\mathrm{h})\right]$, de sorte que $\mathrm{dh} / \mathrm{dt}$ est toujours $<0$ et, comme les joueurs sont homogènes, nous rejoignons de n'importe quel point du jeu l'attracteur unique $B$. réponse aux stratégies de qualité haute des autres joueurs, même pour des vignobles qui pourraient prétendre produire des vins de haute qualité. Sur un marché à producteurs nombreux et coûts d'information élevés, la qualité du vin aristocratique est difficile à connaître et le prix n'est pas un indicateur suffisant ( $c f$. Gergaud, 2000 pour le cas du Champagne). On peut imaginer que l'offre pouvant contenir, sans possibilité de discrimination nette, de la qualité et de la nonqualité, les consommateurs sont amenés à acheter de façon aléatoire, avec la même disposition à payer du fait de leur ignorance de la qualité et donc au même prix, des vins qui se révèleront dans certains cas bons et dans d'autres médiocres. Les différents vins sont alors vendus à des prix proches et la stratégie opportuniste de diminution de la qualité est gagnante en économisant sur les coûts.

L'imperfection de l'information peut aussi conduire à ce que la prime d'alignement vers le bas $\left(\mathrm{a}_{2}\right)$ soit positive, c'est-à-dire que le coût de maintenir de la qualité haute en réponse à l'offre de qualité basse des autres offreurs au lieu de diminuer aussi celle-ci soit positif. Cela sera le cas si le vendeur ne peut être crédible et signaler sa qualité particulière. L'imperfection de l'information induit alors des stratégies opportunistes qui réduisent la qualité et créent un grand risque d'expulsion du domaine des marchés de vins aristocratiques. Comme le seul EES est un équilibre bas, la seule SES une stratégie de faible qualité, tous les producteurs, après observation des gains et apprentissage, s'alignent sur la stratégie basse. Le fonctionnement du marché est par lui-même incapable de modifier cet équilibre bas et seuls des changements dans les conditions institutionnelles de l'information et/ou de la production peuvent le faire.

Un tel type de configuration peut représenter de façon pertinente des marchés particuliers. Il peut également modéliser un moment du fonctionnement de certains marchés. On peut en effet observer dans l'histoire des marchés des vins différents 
cas de situations de crise (notamment dues à de la sur ou sous-production) qui génèrent des comportements opportunistes très attractifs ( $c f$. pour le cas du Champagne, Barrère, 2003). L'incitation à « tricher » sur la qualité devient particulièrement forte et l'est d'autant plus que les offreurs ont intérêt à être parmi les premiers à tricher dans la mesure où cette stratégie est la plus payante au début. En revanche l'institutionnalisation de la triche comme comportement détruit en fin de compte le marché.

Ce sentier $2 \mathrm{a}$ est donc un sentier qui conduit à sortir des marchés aristocratiques. On peut en rapprocher le cas de vins qui ont, à un moment de l'histoire, fait partie des segments haut de gamme et qui aujourd'hui les ont quittés, comme certains vins de la vallée de la Loire, ou encore la Clairette de Die, et, plus largement, nombre de vins blancs effervescents qui auraient pu constituer des rivaux potentiels du Champagne, mais n'ont pas su mettre en place les politiques de qualité de ce dernier (et les changements institutionnels correspondants).

\section{La configuration II et le sentier $2 b$ de sortie}

La configuration II est obtenue quand $\mathrm{a}_{1}$ et $\mathrm{a}_{2}$ sont positifs ; la meilleure réponse à $\mathrm{H}$ est $\mathrm{h}$, ce qui est favorable, comme dans la configuration I, à la poursuite d'une politique de qualité mais la meilleure réponse à $\mathrm{B}$ est $b$, ce qui favorise les politiques de faible qualité et nous éloigne de la configuration I. Le modèle montre qu'existent deux EES, E1 $(h, H)$ et E2 (b,B), chacun ayant un bassin d'attraction contigu et séparé en $\lambda=\frac{a_{2}}{a_{1}+a_{2}}$. Cela conduit à une qualité aléatoire.

Pour avoir $\mathrm{a}_{1}>0$, il faut que les consommateurs puissent discriminer entre les vendeurs, reconnaître les vendeurs de qualité haute et ceux de qualité basse, par exemple par le biais de marques ou du fait de réputations, et rémunérer ainsi l'offre de qualité haute. Les offreurs sont de ce fait incités à proposer de la qualité élevée quand leurs concurrents font la même chose, pour éviter d'être éliminés du marché et d'obtenir les gains plus importants attachés à la qualité haute. Cependant, pour avoir simultanément $\mathrm{a}_{2}>0$, s'aligner sur la qualité basse quand elle est jouée par les autres, il faut que l'information ne permette pas de se singulariser suffisamment en vendant de la bonne qualité. C'est ce qui se produit quand la vente par des concurrents, sur le même marché, de produits de basse qualité crée une externalité négative en matière de qualité du fait de l'imperfection de l'information. Les consommateurs sont dubitatifs si bien que la stratégie de qualité élevée n'est rentable que si elle est collectivement jouée. Cela peut correspondre à une situation dans laquelle les producteurs ont un nom (de sorte que quand la qualité jouée par les autres est haute ils s'alignent sous peine d'être sanctionnés) mais un nom à la réputation insuffisante pour le mettre à l'écart de la suspicion créée quand des joueurs proposent de la qualité dégradée. L'offreur ne peut, seul, renverser la situation; il ne peut risquer de maintenir sa qualité en présence de qualité dégradée qui rend les consommateurs suspicieux. Une telle situation est caractéristique d'un régime de marque tel que celle-ci permet d'isoler les mauvais producteurs quand les autres sont bons mais n'est pas (encore) assez forte pour sauver les bons offreurs quand les autres sont mauvais. Les externalités négatives sont plus fortes que les externalités positives et empêchent d'assurer qu'une segmentation claire entre haute et basse qualité s'établisse et se reproduise.

Dans de telles situations, comme le montre la dynamique du modèle, la qualité est imprévisible. Elle passe d'un niveau élevé à un niveau faible, en fonction des chocs stochastiques et des conditions conjoncturelles. Un marché aristocratique, qui nécessite une qualité soutenue et régulière, ne peut se reproduire sur ces bases. Si rien n'intervient dans les conditions du jeu l'évolution tend à exclure ce marché du domaine aristocratique, le sentier de sortie étant ici dénommé $2 b$. 
Les configurations II et III : un exemple de réponse institutionnelle par l'AOC

Le modèle, en précisant tous les cas de figure possibles, nous permet de voir comment un changement des conditions institutionnelles, c'est-à-dire ici des règles du jeu, peut initier un nouveau sentier de développement. La sortie dans la configuration III est liée au fait que $\mathrm{G}(\mathrm{h}, \mathrm{H})<\mathrm{G}(\mathrm{b}, \mathrm{H})$ et $\mathrm{G}(\mathrm{b}, \mathrm{B})>\mathrm{G}(\mathrm{h}, \mathrm{B})$, de sorte que les stratégies basses sont dominantes et dans la configuration II au fait que $\mathrm{G}(\mathrm{h}, \mathrm{H})>\mathrm{G}(\mathrm{b}, \mathrm{H})$ mais que $\mathrm{G}(\mathrm{b}, \mathrm{B})>\mathrm{G}(\mathrm{h}, \mathrm{B})$, si bien qu'en cas de stratégie basse des autres offreurs la meilleure réponse est de s'aligner sur elle. Reproduire la segmentation aristocratique en restant sur les segments aristocratiques implique de modifier les signes de $\mathrm{a}_{1}$ et $\mathrm{a}_{2}$ pour obtenir les conditions de la configuration I, h devenant la stratégie dominante.

La modification des conditions institutionnelles peut concerner les conditions d'information et/ou les conditions de production. Faire de h la meilleure réponse à $\mathrm{H}$ et à $\mathrm{B}$ implique de pouvoir discriminer strictement entre les offreurs, de pouvoir séparer radicalement aux yeux des consommateurs bons et mauvais producteurs. Dans les situations de crise, quand l'attrait des comportements opportunistes enfle brusquement parce que chacun cherche à « sauver sa peau », il convient en outre pour les consommateurs d'avoir une connaissance immédiate de cette différence, ce que ne permet pas nécessairement le jeu de la réputation, l'intérêt à court terme de brader sa réputation l'emportant sur l'intérêt à long terme de la reproduire (Shapiro, 1982). Pour les mêmes raisons, des solutions purement privées sont insuffisantes, chacun ne pouvant se porter garant de lui-même de façon crédible. C'est pourquoi apparaissent des solutions collectives : labels, certifications, .... Cependant, la modification des conditions d'information conduit à s'intéresser aux conditions de production. $\mathrm{La}$ connaissance de la qualité par une autorité collective peut se fonder sur un contrôle ex-post de la qualité (le certificateur goûte le produit et accorde ou non son sceau). Quand le risque d'opportunisme est fort, ce contrôle peut se révéler insuffisant car le marché a besoin d'une information immédiate et sûre. D'où le recours à l'autre forme de contrôle, le contrôle ex-ante : la certification ne sera accordée qu'à ceux qui respectent un cahier des charges explicite et des organismes de gestion de la certification seront mis en place. Dans le cas du Champagne par exemple, l'instauration des premières formes de contrôle collectif qui allaient aboutir à la législation de l'Appellation d'origine contrôlée (AOC) pour les vins s'est manifestée par l'affichage sur les lieux de vinification du nom des vignerons qui ne respectaient pas les normes de production (notamment en utilisant du raisin extérieur à la zone de délimitation ou en ne respectant pas les règles de conduite de la vigne) ainsi que de l'affichage public du nom des « négociants fraudeurs ». La fixation d'un cahier des charges peut en outre être l'occasion, comme cela s'est révélé être le cas très souvent, de durcir les normes de production pour élever la qualité livrée au marché et positionner ainsi le vin vers le haut.

L'AOC apparaît alors comme une gestion institutionnelle du marché, dans des situations de type configurations II et III, afin d'imposer une politique de qualité et d'assurer le maintien des producteurs dans la catégorie des vins aristocratiques. Dans le cas du Champagne, une telle organisation a été particulièrement utile dans les périodes de difficultés, quand les préoccupations de court terme tendaient à l'emporter sur celles de long terme. L'AOC a exclu du jeu les stratégies d'opportunisme et de passager clandestin, a été le moyen d'une élévation continue des standards de qualité, a permis d'élargir l'écart entre Champagne et autres vins blancs effervescents et, en fin de compte, a réussi à positionner le Champagne comme le seul vin effervescent de luxe. L'AOC, contrainte pour les producteurs et garantie de qualité 
pour les consommateurs, produit de la qualité.

Une gestion rigoureuse de l'AOC accroît $\mathrm{a}_{1}[\mathrm{G}(\mathrm{h}, \mathrm{H})-\mathrm{G}(\mathrm{b}, \mathrm{H})]$ parce qu'un producteur de mauvaise qualité peut perdre son droit à l'appellation. En même temps $\mathrm{a}_{2}[\mathrm{G}(\mathrm{b}, \mathrm{B})-$ $\mathrm{G}(\mathrm{h}, \mathrm{B})]$ demeure positif aussi longtemps que l'AOC permet de signaler les bons producteurs. Sur les marchés correspondant à la configuration II, la probabilité (1- $\lambda)$ d'un équilibre optimal $(\mathrm{h}, \mathrm{H})$ est une fonction croissante de $\mathrm{a}_{1}$ et une fonction décroissante de $\mathrm{a}_{2}{ }^{5}$. Pour $\mathrm{a}_{1}$, la prime d'alignement sur la qualité haute (i.e le supplément de profit à produire de la haute qualité plutôt que de la basse quand les autres vendent de la bonne qualité) deux effets opposés existent. Le premier est un effet de réputation qui tend à élever $a_{1}$. Une gestion plus rigoureuse de l'AOC consolide la réputation des vendeurs de bonne qualité. Le second est un effet d'opportunisme, fournir de la mauvaise qualité afin d'exploiter la bonne image du vin créée par les stratégies de qualité haute des concurrents sans en payer le prix. L'AOC interdit la qualité basse ou en augmente le prix en faisant peser un risque de perte du bénéfice de l'appellation. Quant à la valeur de $\mathrm{a}_{2}$, prime d'alignement sur la basse qualité, elle tend à diminuer sur un marché qui comprend de plus en plus d'offreurs identifiables. L'AOC produit une discrimination croissante au sein des producteurs et une corrélation croissante entre gains et qualité effective. Sur des marchés à grand nombre de petits producteurs les investissements requis par une politique de marque sont trop élevés pour ceux-ci. $A$ contrario, la stratégie d'AOC consolide leur croissance et apparaît comme un nouveau bien de club (Torre, 2002 ; Stanziani, 2004). Elle ouvre un nouveau type de sentier de développement, un sentier 3 de gestion institutionnelle de la qualité, avec une forme $3 \mathrm{a}$ dans le cas des marchés de type configuration II et une forme 3 b dans celui de ceux de

5. $1-\lambda\left(=a_{1} /\left(a_{2}+a_{1}\right)\right.$ i.e $1 /\left(1-(\lambda)=1+\left(a_{2} / a_{1}\right)\right.$. type configuration III. En revanche, la voie de l'exclusion du marché aristocratique reste ouverte si la gestion de l'AOC est laxiste et n'assure pas une qualité suffisamment forte et régulière (sentiers $2 \mathrm{c}$ et $2 \mathrm{~d}$ ), conduisant in fine au même résultat que les sentiers de sortie directe, $2 \mathrm{a}$ et $2 \mathrm{~b}$, des configurations II et III $^{6}$.

\section{La configuration IV : sa complexité et les divers sentiers correspondants}

Elle correspond à $\mathrm{a}_{1}$ et $\mathrm{a}_{2}<0$; b est, comme dans la configuration III, et contrairement à la « bonne » configuration I, la meilleure réponse à $\mathrm{H}$, ce qui est le cas si l'effet d'opportunisme est supérieur à l'effet de réputation individuelle; de façon quelque peu contradictoire, $h$ est la meilleure réponse à $\mathrm{B}$.

Si nous cherchons les caractéristiques de l'information susceptibles de conduire à un tel résultat, nous observons qu'elles semblent difficilement compatibles. Elles tendent plutôt à se contredire, en impliquant que l'information soit simultanément assez bonne pour satisfaire la condition 2 et suffisamment mauvaise pour satisfaire la condition 1. Celle-ci signifie en effet que le producteur opportuniste peut maintenir une stratégie de mauvaise qualité quand les autres jouent la qualité tandis que celle-là dit qu'il peut signaler sa spécificité dans un monde de qualité médiocre et choisir alors une qualité élevée. La présence de marchés aristocratiques correspondant à cette configuration IV ne peut s'expliquer, s'ils existent, par des conditions particulières d'information mais, de façon plus convaincante, comme résultant d'un type donné de préférences des consommateurs. Encore faut-il distinguer entre deux types de situations d'offre. Les producteurs peuvent former une population homogène, ce qui est le cas quand leurs différences ne suffisent pas à engendrer des réponses différentes à des

6. Les différents sentiers sont récapitulés dans l'Annexe 3. 
situations identiques. Ils peuvent au contraire former une population hétérogène, par exemple parce que des chocs aléatoires ou des mutations ont accru la diversité entre producteurs, et répondre alors différemment dans des contextes pourtant identiques. La théorie des jeux évolutionnaires montre que les jeux se déroulent différemment et aboutissent à des résultats différents selon que les joueurs sont ou non homogènes.

\section{Des joueurs homogènes}

Il existe alors un seul EES avec une qualité $\lambda \mathrm{h}+(1-\lambda) \mathrm{b}$. Cet équilibre peut prendre deux formes. Dans la première, tous les producteurs proposent une qualité intermédiaire. S'agit-il d'une situation susceptible de se concrétiser?

Nous pouvons constater que tous les marchés aristocratiques ne sont pas pour autant des marchés de vins aussi réputés que ceux de la Romanée-Conti ou du Pétrus. Pour une consommation courante, même s'il s'agit de consommation aristocratique, les consommateurs peuvent préférer une qualité intermédiaire à un prix intermédiaire. Comme les producteurs sont similaires ils offrent tous de la qualité intermédiaire. Encore faut-il que le marché arrive à s'adapter au niveau de qualité et de prix souhaité, ce qui pose la question de l'autorégulation marchande.

Si la qualité fournie est trop élevée par rapport au niveau de qualité demandé, la valeur absolue de $a_{1}$ (qui est négatif) augmente tandis que celle de $\mathrm{a}_{2}$ (qui est aussi négatif) décroît, de sorte que $\lambda$ et le niveau de qualité offert diminuent aussi. Si, à l'inverse, le niveau offert est inférieur au niveau demandé, $\mathrm{a}_{1}$ (qui est négatif) diminue en valeur absolue et $\mathrm{a}_{2}$ (également négatif) accroît sa valeur absolue, de sorte que $\lambda$ et la qualité s'élèvent. D'un point de vue économique ce processus implique que l'information sur les préférences soit transmise au marché par le mécanisme de prix et que les producteurs puissent répondre dans les temps. Les conditions économiques réelles du marché considéré peuvent ne pas satisfaire les conditions formelles requises pour rester dans cette configuration aristocratique. De ce fait l'on peut identifier un premier sentier d'évolution 2e : les producteurs ne peuvent ajuster la qualité produite aux désirs des consommateurs, la qualité est insuffisante pour que le vin continue à appartenir au marché aristocratique, les producteurs perdent leur réputation, et il y a sortie du marché aristocratique. Un deuxième sentier est le sentier $3 \mathrm{c}$ : une solution institutionnelle, de type AOC, permet de rester dans le marché aristocratique ou d'y retourner, en conduisant à un ajustement de la qualité et en garantissant la reproduction de ce niveau. Un autre sentier, nouveau, le sentier 4, traduit un ajustement de marché ; les producteurs offrent la qualité intermédiaire correspondant aux préférences des consommateurs.

Une seconde possibilité est que tous les producteurs fournissent en proportion $\lambda \mathrm{du}$ vin de haute qualité et en proportion $(1-\lambda) \mathrm{du}$ vin de basse qualité. La condition $\mathrm{a}_{1}, \mathrm{a}_{2}<0$ implique : $\mathrm{G}(\mathrm{h}, \mathrm{H})$ et $\mathrm{G}(\mathrm{b}, \mathrm{B})<\mathrm{G}(\mathrm{b}, \mathrm{H})$ et $\mathrm{G}(\mathrm{h}, \mathrm{B})$; c'est-à-dire qu'une combinaison de stratégies de qualité identiques rapporte moins qu'une combinaison de stratégies différentes. Une telle situation peut correspondre à un marché sur lequel la coexistence de deux qualités, $\mathrm{H}$ et $\mathrm{B}$, désormais distinguables, élargit la demande du fait de la diversité des dispositions à payer. Au lieu d'avoir une concurrence forte sur un marché étroit, comme dans les situations $(\mathrm{h}, \mathrm{H})$ mais aussi (b,B), des offres complémentaires permettent de satisfaire davantage de consommateurs et diminuent les pressions concurrentielles. Dans ces conditions, une nouvelle différenciation apparaît sur le segment aristocratique et nous avons un nouveau sentier de développement, le sentier 5 , sentier de différenciation aristocratique. C'est par exemple le cas des seconds vins ; si le «premier» vin a un nom célèbre, symbole de qualité aristocratique, le second profite de sa réputation comme garantie de 
qualité, même s'il est lui-même reconnu comme de qualité inférieure, et il satisfait un nouveau type de demande.

Cependant, les conditions économiques de fonctionnement du marché peuvent empêcher son autorégulation. Des producteurs peuvent offrir une qualité inférieure au niveau exigé par les consommateurs même pour la qualité basse, perdre leur réputation, et être expulsés du marché aristocratique (sentier 2f). La gestion des systèmes d'AOC, comme plus haut, peut être un bon moyen d'ajuster en qualité offres et demandes (sentier 3d). Enfin, les marchés aristocratiques peuvent mêler production de qualité intermédiaire et production de différentiation et évoluer selon le sentier 6 .

\section{Des joueurs hétérogènes}

$\mathrm{Si}$ la population des producteurs se répartit en deux types différents de joueurs, le jeu donne deux EES, opposés et tous deux asymétriques, $(\mathrm{h}, \mathrm{B})$ et $(\mathrm{b}, \mathrm{H})$. Certains producteurs se spécialisent dans la production de qualité haute et d'autres de qualité basse. Cela peut correspondre à un marché incluant deux niveaux différents de qualité, un haut et un bas, et à un sentier $5 b$, similaire au précédent sentier $5 \mathrm{a}$, propre à la variante homogène. Comme précédemment, les conditions économiques peuvent empêcher le jeu de la régulation marchande. Des producteurs peuvent être contraints à quitter le marché, faute d'un niveau suffisant de qualité, y compris pour servir la tranche basse du marché (sentier 2g); l'AOC peut encore être un bon moyen d'ajuster en qualité offres et demandes (sentier 3e).

\section{Conclusion}

Notre modèle évolutionnaire de détermination stratégique de la qualité permet de définir l'ensemble des sentiers possibles de développement pouvant caractériser les marchés aristocratiques des vins. Les données économiques sont toutes rassemblées dans la considération de seulement deux déter- minants, les primes d'alignement stratégique sur la haute et la basse qualité. Nous disposons ainsi d'un outil qui peut aider à l'analyse des mutations actuelles des marchés viticoles en considérant l'évolution de la forte segmentation qui les caractérisait.

Une nette amélioration de la qualité sur les segments bas fait que les marchés de masse concurrencent plus directement certains marchés autrefois protégés. Les nouveaux pays producteurs sont capables de produire à grande échelle des vins de qualité standard mais régulière et ce à un coût inférieur à celui des vieux pays du vin. En même temps, la demande dans ces pays tend à diminuer, les habitudes de consommation changent (Anderson, 2004). Les producteurs tentent de réagir à ces mutations en multipliant le recours aux AOC et en s'orientant plus nettement vers l'augmentation de la qualité, ce qui tend à dévaloriser le signe de qualité que pouvait représenter l'AOC quand elle ne concernait qu'une minorité de vins.

Cependant, derrière ces diverses modifications, gît une mutation plus fondamentale. L'ancienne segmentation des marchés viticoles reposait sur un paradigme aristocratique du goût qui se trouve aujourd'hui remis en cause. Il cède la place à un nouveau paradigme, celui du goût marchand. Le paradigme sociétal représente en effet aujourd'hui la société comme une société démocratique et marchande, marquée par la concurrence et la mobilité sociales. L'égalité de principe des individus est inscrite dans les Constitutions. La segmentation sociale est modifiée : seule une petite élite se fait remarquer, la jet set, quantitativement très restreinte et définie au niveau international ; les classes dominantes deviennent discrètes; le mode de vie du reste de la population se rapproche, les inégalités se dissimulent derrière des similitudes formelles et les distinctions très fortes du passé s'estompent dans la société de masse. Tous les individus sont formellement égaux dans des relations de marché ou de contrat. 
De ce fait, tout le monde a droit au luxe et au bon goût, à condition de payer, c'est-àdire de respecter la contrainte marchande. Certes, comme les multiples études empiriques du courant de la distinction l'ont montré, la distinction des goûts reste forte dans certains domaines, notamment dans ceux qui exigent un important patrimoine culturel comme celui de l'art. En revanche, l'opposition entre le goût aristocratique, le bon goût, et le goût populaire, assimilé à l'absence de goût, voire au mauvais goût, tend à disparaître. Le goût est devenu l'affaire de tous, les biens de goût, même s'ils connaissent des différenciations, peuvent entrer dans la plupart des consommations et la plupart des biens intègrent désormais des caractéristiques de goût. Dans le domaine du vin, comme dans celui de la gastronomie, et plus généralement dans celui des industries du luxe, si demeurent des segments élitaires, occupés par des super-stars (les restaurants trois étoiles, dans lesquels les additions moyennes dépassent aujourd'hui les 200 euros par personne ou les premiers crus réputés dont les bouteilles dépassent allègrement les 50 euros au Château et les 200 euros au restaurant), le luxe marchand devient accessible à nombre de foyers à travers le monde. Cela se traduit par le fait que, contrairement aux apparences et même s'ils gardent une image de rareté ou « d'extra-ordinaire », nombre de biens de luxe sont produits en grande quantité. Vuitton vend des dizaines de milliers de certains modèles de sacs et en produit chaque année plus de trois millions. Hermès vend annuellement plus de 50000 exemplaires de ses modèles de base de chemises. Le secteur du Champagne produit et vend plus de 300 millions de bouteilles. C'est dire que la demande de biens de luxe devient une demande de masse. Elle s'alimente de l'accroissement du revenu de couches de plus en plus larges de la population mondiale. En 2004, selon les rapports annuels de Merrill Lynch et de Capgemini, 8,3 millions de ménages auraient disposé d'un capital financier mobilisable (exclusion faite de la valeur de leurs entreprises ou de leur immobilier) supérieur à 1 million de dollars. Selon le dernier rapport Goldman Sachs, dès 2003, plus de 45 millions de Chinois avaient un pouvoir d'achat supérieur à 30000 dollars et le rapport du cabinet Ernst \& Young prévoit une croissance des ventes de biens de luxe en Chine de $20 \%$ par an sur la période 2005-2008 et de $10 \%$ minimum sur la période 2009-2015. De plus, les consommateurs réguliers de biens de luxe se voient accompagnés de très nombreux consommateurs occasionnels de tels biens, ce qui contribue, vu leur nombre, à constituer une demande de masse.

Un paradigme marchand du goût se met en place, paradigme qui mine lentement mais sûrement l'ancienne organisation des marchés. Les marchés viticoles sont profondément affectés par ce mouvement de fond. Les marchés aristocratiques voient leurs sentiers habituels de développement fragilisés. La formalisation présentée, après avoir explicité les conditions de la reproduction de la segmentation aristocratique, devrait pouvoir éclairer ces changements en repérant les éléments qui jouent sur les deux déterminants synthétiques relevés, les primes stratégiques d'alignement sur les qualités basse et haute.

L'auteur remercie les deux referees anonymes dont les remarques, approfondies et détaillées, ont permis d'améliorer sensiblement la version initiale de l'article. II demeure naturellement seul responsable des imperfections du texte. 


\section{RÉFÉRENCES BIBLIOGRAPHIQUES}

Akerlof G. (1970). The Market for "Lemons": Quality Uncertainty and the Market Mechanism. In Quarterly Journal of Economics, $\mathrm{n}^{\circ}$ 84(3), p. 488-500.

Anderson K. (Ed.) (2004). The World's Wine Markets. Globalization at Work. Cheltenham, Edward Elgar.

Barrère C. (2003). Un processus évolutionnaire de création institutionnelle d'une convention de qualité : l'histoire exemplaire de la création d'un produit de luxe, le Champagne. Paris, Economie appliquée, 2003/3, p. 133-170.

Barrère C., Santagata W. (2005). La mode, une économie de la créativité et du patrimoine à l'heure du marché. Paris, $L a$ Documentation Française.

Becker G. (1996). Accounting for tastes. Cambridge, Harvard University Press.

Bourdieu P. (1979). La distinction, critique sociale du jugement. Paris, Editions de Minuit.

Caves R. (2000). Creative Industries. Contracts between Art and Commerce. Cambridge, Mass., Harvard University Press.

Chamberlin E. (1933). The theory of monopolistic competition. Cambridge, Cambridge University Press.

Elias N. (1973). La civilisation des mours. Paris, Calmann-Lévy, Édition Presses Pocket.

Fischler C. (1993). L'Homnivore. Paris, Editions Odile Jacob.
Gergaud O. (2000). Fonctions de prix hédonistiques et information imparfaite: le rôle de la réputation sur le marché du vin de Champagne. Thèse pour le doctorat en sciences économiques, Reims, Université de Reims.

Lancaster K.-J. (1966). A new approach to consumer theory. In Journal of Political Economy, 74, p. 132-157.

Levi-Strauss C. (1964). Le cru et le cuit. Paris, Plon.

Shapiro C. (1982). Consumer Information, Product Quality, and Seller Reputation. In Bell Journal of Economics, ${ }^{\circ} 13$, p. 20-35.

Simmel G. (1904). Fashion. In American Journal of Sociology, ${ }^{\circ}$ 62, p. 61-76. Spencer H. (1854). On manners and fashion, Essays on education and kindred subjects. Réédition 1966, London, Dent/Everyman. Stanziani A. (2004). Wine Reputation and Quality Controls: the Origin of the AOCs in $19^{\text {th }}$ Century France. In European Journal of Law and Economics, $\mathrm{n}^{\circ} 18$, p.149-167.

Torre A. (2002). Les AOC sont-elles des clubs ? Réflexions sur les conditions de l'action collective localisée, entre coopération et règles formelles. In Revue d'Économie Industrielle, $\mathrm{n}^{\circ} 100,3^{\circ}$ trimestre, p. 39-62.

Veblen T. (1899). Théorie de la classe de loisir. Traduction française, Paris, Gallimard, 1970.

Weibull J.-G. (1996). Evolutionary Games Theory. Cambridge, MIT Press. 


\begin{abstract}
ANNEXES
Annexe 1. Les équilibres évolutionnaires

La théorie des jeux évolutionnaires s'intéresse aux équilibres représentés par des ensembles dans lesquels tous les joueurs utilisent des stratégies évolutionnairement stables, c'est-àdire résistantes à toute invasion d'une stratégie alternative [Weibull 1996]. Si la stratégie est évolutionnairement stable, dans une population donnée, cela signifie qu'elle rapporte davantage, face à toute invasion, que la stratégie mutante. En appelant $\varepsilon$ le pourcentage de la population mutante, $\varepsilon \in(0,1)$, I la stratégie (pure ou mixte) évolutionnairement stable et $\mathbf{J}$ la stratégie mutante, l'on a :

$$
\mathrm{E}\{\mathrm{G}(\mathrm{I}, \varepsilon \mathrm{J}+(1-\varepsilon) \mathrm{I})\}>\mathrm{E}\{\mathrm{G}(\mathrm{J}, \varepsilon \mathrm{J}+(1-\varepsilon) \mathrm{I})\} .
$$
\end{abstract}

Une stratégie I est évolutionnairement stable si cette inégalité vaut pour toute stratégie $\mathrm{J} \neq \mathrm{I}$.

L'on montre qu'une stratégie peut être qualifiée d'évolutionnairement stable si, face à l'invasion d'une stratégie autre $\mathrm{J}$, pour tout joueur $\mathrm{i}=\{1, \ldots \mathrm{n}\}$ et $\forall \mathrm{J} \subset\{\mathrm{S}\}$, ensemble des stratégies, les deux conditions suivantes sont réunies :

(1) $\mathrm{E}\{\mathrm{Gi}(\mathrm{I}, \mathrm{I})\} \geq \mathrm{E}\{\mathrm{Gi}(\mathrm{J}, \mathrm{I})\}$

(2) ou (2a) est vrai ou (2b) est vrai

(2a) $\mathrm{E}\{\mathrm{Gi}(\mathrm{I}, \mathrm{I})\}>\mathrm{E}\{\mathrm{Gi}(\mathrm{J}, \mathrm{I})\}$

(2b) $\mathrm{E}\{\mathrm{Gi}(\mathrm{I}, \mathrm{J})\}>\mathrm{E}\{\mathrm{Gi}(\mathrm{J}, \mathrm{J})\}$

On définira donc des stratégies évolutionnairement stables (SES) et des équilibres évolutionnairement stables (EES). Dans une population homogène, les EES correspondront à l'adoption par tous les joueurs d'une stratégie évolutionnairement stable (pure ou mixte), tandis que dans les populations hétérogènes (polymorphes) ils correspondront à une répartition donnée et stable des sous-groupes jouant chacun une stratégie déterminée.

L'étude évolutionnaire des jeux se fait au moyen de l'hypothèse selon laquelle des stratégies qui rapportent plus que d'autres se voient progressivement préférées par les acteurs. Pour cela l'on considère habituellement un mécanisme de réplication qui associe la variation de la proportion des joueurs recourant à une stratégie et l'écart entre le gain de cette stratégie et le gain moyen obtenu par les autres stratégies.

Nous nous limiterons habituellement à des jeux dans lesquels l'ensemble des stratégies se compose de l'ensemble des stratégies mixtes fondées sur deux stratégies pures, S1 et $\mathrm{S} 2$, et utiliserons un mécanisme standard tel que, en appelant qi la proportion des joueurs choisissant la stratégie I, dqi/dt = qi. [G(I,S) - G(S,S)], S représentant toute stratégie générale du type $[\mathrm{pS} 1,(1-\mathrm{p}) \mathrm{S} 2], 0 \leq \mathrm{p} \leq 1$. 


\section{Annexe 2. La symétrie du jeu}

Pour savoir si une situation peut être représentée par un jeu symétrique, il convient de considérer, non les niveaux de gain mais la structure de ces gains. Si leur structure est identique pour chaque joueur, c'est-à-dire s'ils sont ordonnés de la même façon, le jeu peut être transformé en jeu symétrique. Ainsi, si dans le jeu 1 ci-dessous, l'ordre entre a', b', c', d' est toujours le même que l'ordre entre a, b, c, d nous pouvons par une suite de transformations linéaires (décrites par le passage du jeu 1 au jeu 7) le transformer en jeu 7 symétrique comme décrit ci-dessous, avec $\mathrm{x}=\mathrm{a}_{1} / \mathrm{b}_{1}, \mathrm{y}=\mathrm{a}_{2} / \mathrm{b}_{2}$.

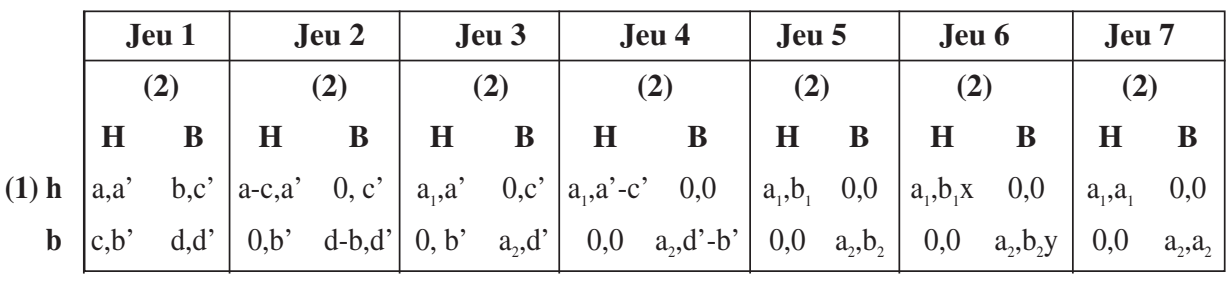

Dans le dernier, le jeu symétrique 7, les équilibres ne sont pas les mêmes que dans le premier, du fait des transformations linéaires effectuées, mais la configuration des équilibres (nombre, types, ...) est la même. Quand $\mathrm{a}_{1}=\mathrm{a}-\mathrm{c}$ est positif (négatif), $\mathrm{b}_{1}=\mathrm{a}^{\prime}-\mathrm{c}^{\prime}$ est aussi positif (négatif), c'est-à-dire que $h$ est la meilleure réponse à $H$ (respectivement b), pour tous les joueurs ; quand $\mathrm{a}_{2}=\mathrm{d}-\mathrm{b}$ est positif (négatif), $\mathrm{b}_{2}=\mathrm{d}^{\prime}-\mathrm{b}$ ' est aussi positif (négatif), c'est-à-dire que b (respectivement $h$ ) est la meilleure réponse à $B$, pour tous les joueurs. Alors, $\mathrm{x}=\mathrm{a}_{1} / \mathrm{b}_{1}, \mathrm{y}=\mathrm{a}_{2} / \mathrm{b}_{2}>0$, et aucune modification de signe n'intervient du jeu 1 au jeu 7, aucun changement dans les configurations d'équilibre. Comme nous privilégions l'étude de ces configurations, la transformation est permise. 


\section{Annexe 3. Les sentiers de développement}

\begin{tabular}{|c|c|c|c|}
\hline \multicolumn{4}{|c|}{ Détermination de la qualité } \\
\hline & $\mathrm{a} 2>0 ; \mathrm{b}=\mathrm{MR}(\mathrm{B})$ & $\mathrm{a} 2<0 ; \mathrm{h}=\mathrm{MR}(\mathrm{B})$ & \\
\hline al $>0 ; \mathrm{h}=\mathrm{MR}(\mathrm{H})$ & $\begin{array}{l}\text { Configuration II } \\
2 \text { EES }=(b, B) \text { et }(h, H) \\
\text { Sentier 2a exit } \\
\text { Sentier 2c exit par régulation } \\
\text { institutionnelle laxiste } \\
\text { Sentier 3a gestion } \\
\text { institutionnelle de la qualité }\end{array}$ & $\begin{array}{l}\text { Configuration I } \\
1 \mathrm{EES}=(\mathrm{h}, \mathrm{H}) \\
\text { Sentier } 1 \text { auto-régulation et } \\
\text { reproduction d'une qualité hau }\end{array}$ & \\
\hline $\mathrm{a} 1<0 ; \mathrm{b}=\mathrm{MR}(\mathrm{H})$ & $\begin{array}{l}\text { Configuration III } \\
1 \mathrm{EES}=(\mathrm{b}, \mathrm{B}) \\
\text { Sentier } 2 \mathrm{~b} \text { exit } \\
\text { Sentier 2d exit par régulation } \\
\text { institutionnelle laxiste } \\
\text { Sentier 3b gestion } \\
\text { institutionnelle de la qualité }\end{array}$ & $\begin{array}{l}\text { Configuration IV } \\
\text { Producteurs homogènes } \\
1 \text { EES }=\lambda \mathrm{h}+(1-\lambda) \mathrm{b} \\
\text { A- Une qualité intermédiaire } \\
\text { Sentier 2e exit par régulation } \\
\text { institutionnelle n'assurant pas } \\
\text { la qualité intermédiaire } \\
\text { Sentier 3c gestion } \\
\text { institutionnelle assurant une } \\
\text { qualité intermédiaire } \lambda \\
\text { Sentier 4, ajustement de } \\
\text { marché à la qualité } \\
\text { intermédiaire } \lambda \\
\text { B- Deux qualités différenciées } \\
\text { Sentier 5a, différenciation par } \\
\text { le marché avec qualité haute et } \\
\text { qualité basse } \\
\text { Sentier 2f exit par régulation } \\
\text { institutionnelle n'assurant pas } \\
\text { la qualité basse } \\
\text { Sentier 3d gestion } \\
\text { institutionnelle assurant une } \\
\text { différenciation des qualités, } \\
\text { haute et basse } \\
\text { C- Super-différenciation : } \\
\text { qualité basse, intermédiaire, } \\
\text { haute } \\
\text { Sentier 6, super- } \\
\text { différenciation, avec qualité } \\
\text { haute, qualité basse et qualité } \\
\text { intermédiaire } \lambda\end{array}$ & \begin{tabular}{|l} 
Producteurs hétérogènes \\
$2 \mathrm{EES}=(\mathrm{h}, \mathrm{B})$ et $(\mathrm{b}, \mathrm{H})$ \\
Sentier $2 \mathrm{~g}$ exit par \\
régulation institutionnelle \\
n'assurant pas la qualité \\
basse \\
Sentier 3e gestion \\
institutionnelle assurant \\
une différenciation des \\
qualités, haute et basse \\
Sentier $5 \mathrm{~b}$, \\
différenciation par le \\
marché avec qualité haute \\
et qualité basse
\end{tabular} \\
\hline
\end{tabular}

